\title{
Microwave Inductive Readout of EBL nanobridge SQUIDs
}

\author{
T. Godfrey, G. Long, J. Gallop, D. Cox, E. Polychroniou, J. Chen, Member, IEEE, E. Romans and L. Hao
}

\begin{abstract}
Niobium-based nanobridge SQUIDs have shown very low noise performance and high frequency operation. We describe how we are bringing together these two aspects of nanobridge SQUIDs with the aim of realising single spin-flip detection using microwave inductive readout techniques, where the nanobridge SQUID is integrated into a superconducting coplanar waveguide (CPW) resonator. Using electron beam lithography (EBL) is ideal for fabricating nanobridge junctions and has the advantage of being easily scalable compared to using a focused ion beam. In this paper, we demonstrate that EBL is suitable for fabricating nanobridge junction SQUIDs, and they exhibit very comparable performance to previous FIB milled SQUIDs. Our integrated devices show potential to be used for flux tunable sensors.
\end{abstract}

Index Terms-SQUIDs, focused ion beam, EBL, nanoscale, superconducting microwave devices, NanoSQUID

\section{INTRODUCTION}

$\mathbf{D}_{\mathrm{s}}$ evelopments in quantum technologies are driving the need to explore and improve the limits of macroscopic quantum superconducting devices such as SQUIDs, already capable of detecting and measuring a wide range of physical parameters, with unequalled sensitivity [1-7]. We are fabricating novel SQUIDs to pursue further improvement, particularly for spinflip detection. Recent advances in nanoscale fabrication have led to further improvement in SQUID sensitivity limits. One of the most notable improvements is the ability to reliably fabricate nanobridge Josephson junctions with dimensions in the 10s of nanometres. The criteria for the nanobridge to display the Josephson effect with a well behaved single valued currentphase relation [8], is its length, $l$, is $<3.5 \xi(T)$ (the Ginzburg Landau coherence length). For pure $\mathrm{Nb}$ at $4.2 \mathrm{~K}, \xi(T)$ is around $40 \mathrm{~nm}$ [9]. The fabrication of consistent nanobridge junctions satisfying these dimensions is challenging and requires focused ion beam milling (FIB) [10-12], or high resolution electron beam lithography (EBL) [9]. Nanobridges junctions have a number of advantages over traditional tri-layer junctions. They can achieve much higher critical currents with much lower capacitance, favouring high frequency operation. Their much

This work was supported by the UK NMS Programme and UCL \& NPL Impact Studentship. (Corresponding author: Ling Hao and T. Godfrey)

L. Hao is with the National Physical Laboratory, Teddington, TW11 0LW, UK (e-mail: ling.hao@npl.co.uk).

Tom Godfrey is with the National Physical Laboratory, Teddington, TW11 OLW, UK and also with London Centre for Nanotechnology, University College London, London WC1H 0AH, UK (e-mail: tom.godfrey@npl.co.uk).

George Long is with the National Physical Laboratory, Teddington, TW11

0LW, UK (e-mail: George.long@npl.co.uk).

John Gallop is with the National Physical Laboratory, Teddington, TW11 0LW, UK (e-mail: john.gallop@npl.co.uk). smaller dimensions are uniquely suited for coupling to local single or clusters of spins. Furthermore, a magnetic moment can be implanted directly on the nanobridge allowing the coupling to single or clusters of spins in an inductive near field regime [13].

Nanobridge SQUIDs have already achieved ultra-low noise performance, Hao et al. have reported FIB nanoSQUIDs with a flux sensitivity of $0.2 \mu \Phi_{0} / \mathrm{Hz}^{1 / 2}$ at $6.8 \mathrm{~K}$ [11]. Moreover, using a dispersive microwave readout instead of the commonly used DC voltage readout can further increase flux sensitivity. Using such a technique Levenson-Falk et al. have reported a flux sensitivity of $30 \mathrm{n} \Phi_{0} / \mathrm{Hz}^{1 / 2}$ at $30 \mathrm{mK}$ [14].

All of our SQUIDs are fabricated from a single $\mathrm{Nb}$ film chosen due to its higher critical temperature and higher magnetic field resilience, in comparison to $\mathrm{Al}$, making it more suitable for electron spin detection experiments at $4.2 \mathrm{~K}$ [15].

In this paper we present the design work and results from our $\mathrm{Nb}$ SQUIDs fabricated with EBL and explore their suitability for future applications employing microwave readout, such as detecting single or clusters of implanted electron spins. We will also outline our work embedding nanobridge SQUIDs into coplanar waveguide (CPW) resonators, leading to SQUID readout in a non-dissipative state to further increase flux sensitivity.

Work on superconducting resonators has become increasingly popular as they are suited to a wide variety of applications and can have an exceptionally high energy resolution. SQUIDs embedded into resonators have already shown practical applications as various quantum sensors and parametric amplifiers $[12,14,16]$. These technologies exploit the non-linear properties associated with the Josephson effect, represented by the Josephson inductance, defined as

$$
L_{\mathrm{J}}=\left(2 \pi \Phi_{0}\right) /\left(I_{0} \cos \delta\right)
$$

where $\Phi_{0}$, the flux quantum, is defined as $h / 2 e$ where $h$ is Planck's constant and $e$ is the charge of an electron. $I_{0}$ is the critical current of the junction and $\delta$ is the phase difference across it. In the case of a dc SQUID with negligible geometric inductance, the Josephson inductance becomes

D. Cox is with the National Physical Laboratory, Teddington, TW11 0LW, UK and also with University of Surrey, Guildford, Surrey, GU2 7XH, UK (e-mail: david.cox@npl.co.uk).

Elias Polychroniou is with the National Physical Laboratory, Teddington, TW11 OLW, UK and also with Imperial College, SW7 2AZ, London, UK (elias.polychroniou@npl.co.uk).

J. Chen is with Department of Mechanical and Aerospace Engineering, Brunel University, Uxbridge UB8 3PH, UK (e-mail: jie.chen@brunel.ac.uk ).

E.J. Romans is with the London Centre for Nanotechnology, University College London, London WC1H 0AH, UK (e-mail: e.romans@ucl.ac.uk). 


$$
L_{\mathrm{S}}=\Phi_{0} /\left(I_{\mathrm{C}} \cos \left(\pi \Phi / \Phi_{0}\right)\right)
$$

where $\Phi$ represents the applied magnetic flux threading the SQUID loop and $I_{\mathrm{C}}$ is the critical current of the device. Equation (2) shows that the SQUID's non-linear inductance is tunable with magnetic flux.

\section{NANOBRIDGE SQUID DESIGN AND RESULTS}

The nanobridge junctions and SQUIDs presented in this work were fabricated using EBL and reactive ion etching (RIE). Using this technique, it is possible to fabricate features with dimensions in the order of a few 10s of nanometres. EBL has certain advantages over using FIB, the etching process causes less damage to the walls of the nanobridges. Also, EBL allows multiple junctions to be fabricated simultaneously, significantly reducing fabrication time. Nominally, all of the nanobridges presented in this paper are designed to have widths of $80 \mathrm{~nm}$ and lengths of $50 \mathrm{~nm}$. After etching the junction widths are typically reduced to around 50-60 $\mathrm{nm}$.

The samples are made on $8 \mathrm{~mm}^{2} \mathrm{Si}$ wafer with a $\sim 150 \mathrm{~nm}$ sputtered layer of $\mathrm{Nb}$. A $200 \mathrm{~nm}$ layer of 950 PMMA A4 is spun on top and baked at $180^{\circ} \mathrm{C}$ for 5 minutes. An electron beam current of $300 \mathrm{pA}$ was used for sub-micron features and $5 \mathrm{nA}$ was used for all larger features, at an acceleration voltage of $100 \mathrm{keV}$. After 30 seconds of development, the exposed regions are reactive ion etched with a flow rate of $35 \mathrm{~cm}^{3} / \mathrm{min} \mathrm{CHF}_{3}$ and $14 \mathrm{~cm}^{3} / \mathrm{min} \mathrm{SF} 6$ for 3 minutes. The remaining resist is removed in acetone at room temperature.

The DC measurement results presented in this paper are from dc SQUIDs with $3 \mu \mathrm{m}^{2}$ loops and approximately $60 \mathrm{~nm}$ wide junctions. All the samples are measured in a $3 \mathrm{~K}$ pulse tube cooler. Fig. 1a \& b characterises the critical current of a
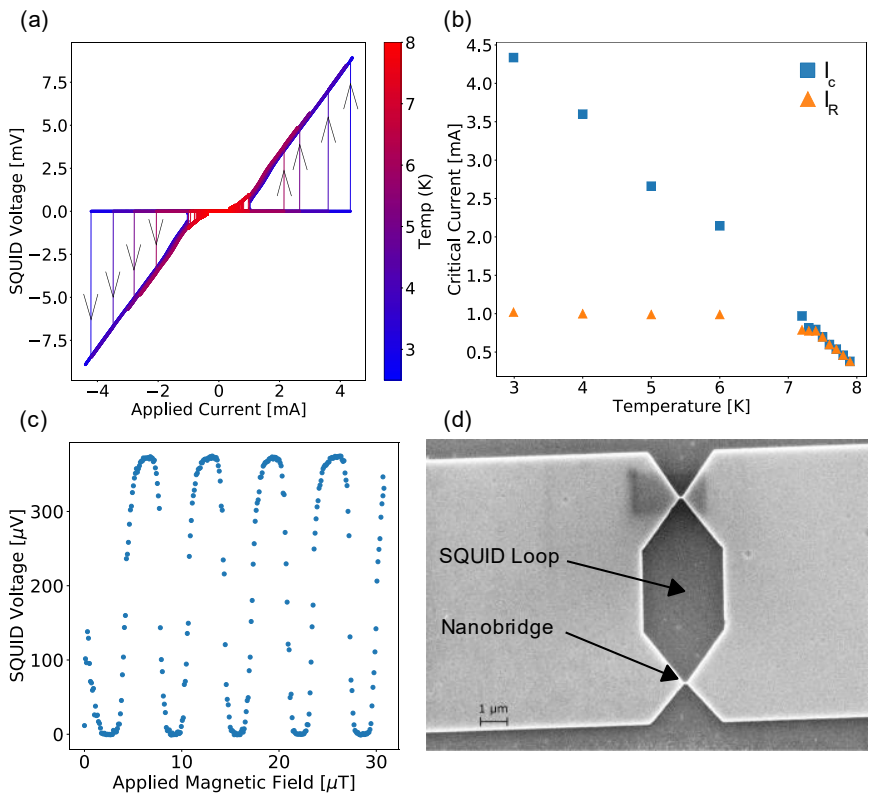

Fig. 1. (a) The current-voltage characteristics for the full temperature range. The current is stepped-up to above the positive critical current and then swept down to below the negative critical current. (b) The critical current of the device $\left(I_{C}\right)$, and the retrapping current $\left(I_{R}\right)$ from 3-7.5 K. (c) The voltage response of the SQUID through 4 periods of $\Phi_{0}$ at $7.6 \mathrm{~K}$ with the device biased at $580 \mu \mathrm{A}$. (d) SEM image of dc SQUID, the lighter regions show the $\mathrm{Nb}$ and the darker region is the Si substrate.
SQUID as a function of temperature. Fig. 1a shows the currentvoltage sweeps over the full range of operating temperatures. At temperatures below $7.2 \mathrm{~K}$ the devices become thermally hysteretic, where the switching current of the up-sweep branch occurs at a higher current than the down-sweep branch, this is due to thermal re-trapping. When the bias current $I_{d c}$ is increased above the critical current $\mathrm{I}_{c}$, a "hot-spot" region in the nanobridge is formed. When $\mathrm{I}_{\mathrm{dc}}$ is then reduced, the hot spot is maintained by Joule heating. The nanobridge returns to the superconducting state when $\mathrm{I}_{\mathrm{dc}}$ is reduced to below the retrapping current $\mathrm{I}_{\mathrm{r}}[17]$. Hysteresis is an issue for conventional (voltage) state measurements, but here the SQUID will be operated in the superconducting state, so thermal hysteresis is not an issue. In this device the thermal re-trapping branch switches back to the superconducting state at approximately $\pm 1 \mathrm{~mA}$, when the temperature is below $6 \mathrm{~K}$. Fig. $1 \mathrm{~b}$ shows the critical current $\left(I_{\mathrm{C}}\right)$, of the up-sweep branches, and the re-trapping current $\left(I_{R}\right)$ extrapolated from Fig. 1a. The modulation of the SQUID output voltage, Fig. 1c, over several periods of $\Phi_{0}$ confirms the devices is a working dc SQUID.

\section{Microwave Readout Resonator Design and Prelim- INARY RESULTS}

To realise superconducting resonators utilising EBL nanobridge SQUIDs, a coplanar waveguide (CPW) geometry was chosen to define the resonant structures and the high frequency feedlines coupling the devices to the input and output ports. A CPW geometry is widely used in superconducting resonators, Fig. 2a shows a representation of a CPW. The design is a standard $50 \Omega$ impedance line with a central conductor width $(W)$ of $10 \mu \mathrm{m}$ and a separation $(S)$ to the ground plane of $5 \mu \mathrm{m}$. The resonator is defined by a coupling capacitor formed by a gap in the central conductor. All the resonators are quarter wavelength $(\lambda / 4)$ geometries terminated to the ground plane through a SQUID, an equivalent circuit is shown in Fig. 2b. The working principles of $\lambda / 4$ resonators are well established within a transmission line model [18].

Our resonators are designed to operate between 4-8 GHz. The resonators are fabricated using the $100 \mathrm{keV}$ EBL with a $5 \mathrm{nA}$ beam current and follows the same process as the dc SQUID fabrication described in section II. A CPW transmission line can be modelled as a distributed inductance with a distributed shunted capacitance, for a superconducting resonator we neglect the resistive element. Using a zero-thickness mapping technique [19] the inductance and capacitance per unit length can be estimated as

$$
\begin{aligned}
& L_{l}=\left(\mu_{0} / 4\right)\left(K\left(k_{0}{ }^{\prime}\right) / K\left(k_{0}\right)\right) \\
& C_{l}=4 \varepsilon_{0} \varepsilon_{e f f}\left(K\left(k_{0}\right) / K\left(k_{0}{ }^{\prime}\right)\right)
\end{aligned}
$$

where $K$ is the complete elliptic integral of the first kind, with $k_{0}=W /(W+2 S)$ and $k_{0}{ }^{\prime}=\sqrt{1-k_{0}^{2}}$. The effective permittivity, $\varepsilon_{e f f}$, accounts for the difference between the permittivity of the substrate and the air. The SQUID loop has a self-inductance, or geometric inductance which we have simulated using the 3DMLSI software package. This can be an important step for understanding the expected flux response in our resonators. In 


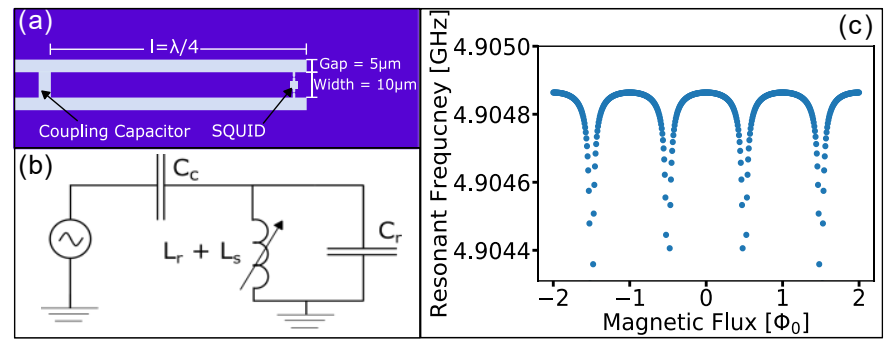

Fig. 2 (a) An illustration of a typical CPW N/4 resonator, coupled with a gap capacitor. The dark blue region is $\mathrm{Nb}$ and the lighter region is the $\mathrm{Si}$ substrate. (b) Equivalent circuit of a CPW, $L_{r}$ and $C_{r}$, with coupling capacitance, $C_{C}$, and SQUID, $L_{s \cdot}$ (c) Simulation of resonant frequency modulation with magnetic flux threading the SQUID loop.
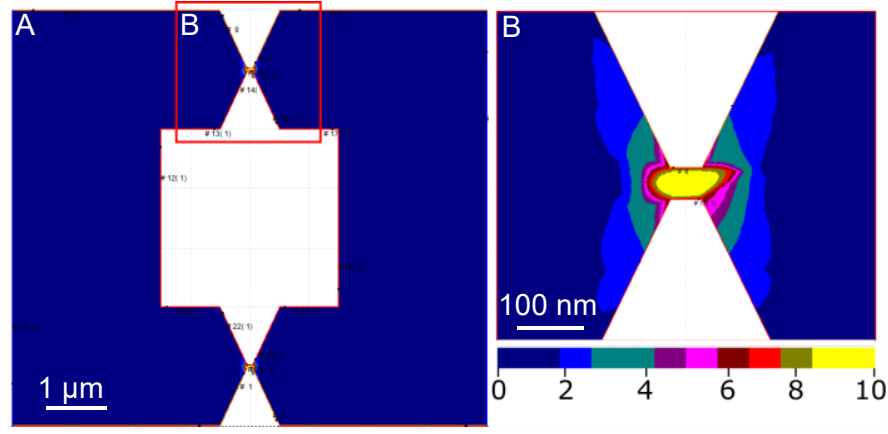

Fig. 3 3D-MLSI simulation of dc SQUID ( $3 \times 3 \mu \mathrm{m}$ loop, $50 \times 50 \mathrm{~nm}$ junction). The simulation maps the normalised density of the current flowing through the superconductor. The current flows from the left-hand side to the right.

Fig. 3 we have simulated a dc SQUID with a loop area of $3 \mu \mathrm{m}^{2}$, and with the typical junction dimensions. The thickness is set to $150 \mathrm{~nm}$ and the mesh size is set to $0.1 \mu \mathrm{m}^{2}$. A simulation result is shown in Fig. 3 which results in an estimation of $2.7 \mathrm{pH}$ for this SQUID geometry.

Inferring from the DC measurements of our dc SQUIDs, section II, we can expect the critical current $\left(I_{\mathrm{C}}\right)$ of the device to be around $4-4.5 \mathrm{~mA}$ at $3 \mathrm{~K}$. This translates to a comparatively small inductive contribution from the Josephson inductance, leading to a very low participation ratio $\left(L_{S} / l_{\mathrm{r}} L_{l}\right)$ where $l_{\mathrm{r}}$ is the resonator length. Using Equation 2 the Josephson inductance is calculated to be $37 \mathrm{fH}$, at zero field, and the inductance of the resonator $\left(l_{\mathrm{r}} L_{l}\right)$ with a length $\left(l_{\mathrm{r}}\right)$ of $6 \mathrm{~mm}$ is estimated to be $2.5 \mathrm{nH}$ using equation 3 . For a single dc SQUID in a $\lambda / 4$ resonator designed for $\sim 5 \mathrm{GHz}$, looking at the results of the simulation shown in (Fig. 2c), the modulation of resonant frequency is low where the SQUID only provides a significant inductance around half flux, where the shift corresponds to an approximately $30 \mathrm{kHz}$ detuning from $\Phi=0$. This had provided the motivation to use arrays of SQUIDs to increase the inductive contribution.

The effect of the temperature dependent kinetic inductance, $L_{l}^{k}$, which is a result of the inertia of Cooper pairs at high frequencies, is considered to be negligible at $3 \mathrm{~K}$ in our resonators. Hence, this added inductance is not considered in our design calculations. For our microwave measurements an SMPM port connects to a copper CPW line on the sample holder which is wire-bonded to the pads of the $\mathrm{CPW}$ resonator on chip. The

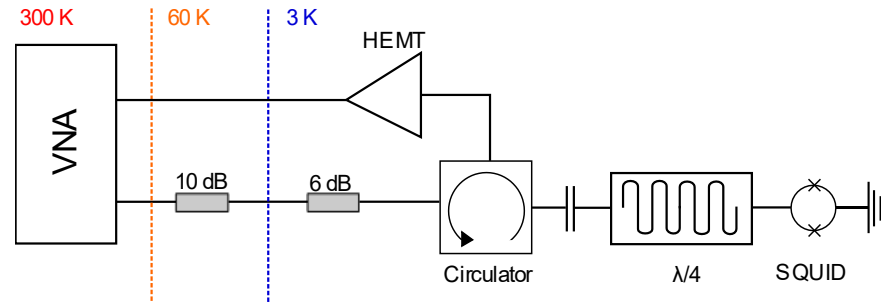

Fig. 4 Schematic of microwave readout circuit used in this experiment. The input line is attenuated to the $3 \mathrm{~K}$ stage.

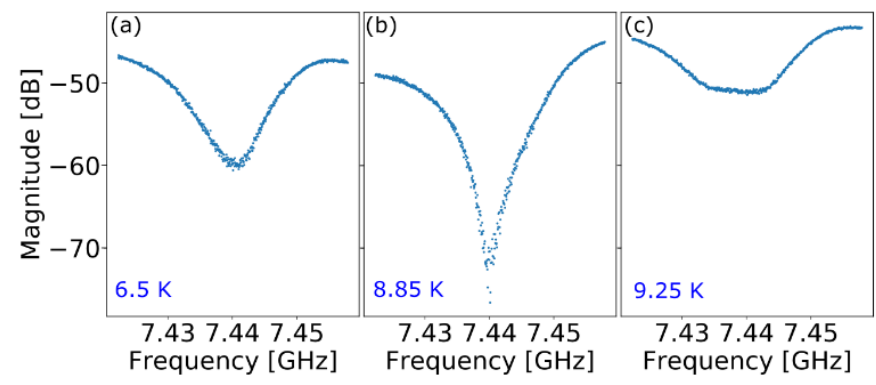

Fig. $5 \mathrm{~S}_{21}$ VNA measurements of a $\mathrm{Nb} \lambda / 4$ resonator terminated with a dc SQUID at: (a) $6.5 \mathrm{~K}$, (b) $8.85 \mathrm{~K}$ and (c) $9.25 \mathrm{~K}$. Measurements where made with a power level of $-67 \mathrm{dBm}$ at the input of the device. The magnitude displayed on the $y$-axis is the response measured at port 2 of the VNA.

output microwave signal is amplified at $3 \mathrm{~K}$ with a low noise high electron mobility transistor (HEMT) and a vector network analyser (VNA) is used to measure the reflected microwave signal, a schematic of the setup is shown in Fig. 4.

The three coupling regimes of our resonators, under, over and critically coupled, are shown in Fig. 5. These three states relate to the matching of the input and resonator impedances. When the resonator is over-coupled, the majority of the resonator's energy is lost through the coupling capacitor. In the regime when the resonator is under-coupled, most of the loss is in the resonator. If the two impedances are equal the resonator output can theoretically become zero.

Our resonators show temperature-dependent coupling. At temperatures below the critical temperature of the $\mathrm{Nb}$ resonator $(\sim 9 \mathrm{~K})$ the resonator is in an over-coupled state (Fig. 5a). When the temperature is increased to very near, but still below, the critical temperature the input and output impedances become near-perfectly matched. At this point the resonator becomes critically-coupled and the minimum dip amplitude is achieved (Fig. 5b). Once the temperature is increased to above the resonator's critical temperature, the resonator becomes far more lossy and becomes under-coupled (Fig. 5c). We attribute this to a perfect impedance match, between the input and the resonator, at this temperature. This could be due to an increase in losses from the kinetic inductance, which is strongly temperature-dependant close to the critical temperature.

\section{CONCLUSIONS}

We have demonstrated that EBL is suitable for fabricating nanobridge junction SQUIDs, and thus far they have proved to be worth pursuing as an alternative to FIB milled SQUIDs. This is encouraging and we wish to perform further measurements to investigate the fabrication limits and low-noise performance 
of these devices. Our $\mathrm{Nb} \mathrm{CPW}$ resonators show potential to be used for flux tunable sensors, however, there is still fundamental work to be done to resolve a frequency shift in a tunable SQUID that has such a high critical current. We are motivated to investigate multiple SQUIDs in a single resonator to increase the inductive participation ratio.

\section{REFERENCES}

[1] C. P. Foley and H. Hilgenkamp, "Why NanoSQUIDs are important: an introduction to the focus issue," Superconductor Science and Technology, vol. 22, no. 6, p. 064001, 2009/05/14 2009.

[2] C. Granata and A. Vettoliere, "Nano Superconducting Quantum Interference device: A powerful tool for nanoscale investigations," Physics Reports, vol. 614, pp. 1-69, 2016/02/19/2016.

[3] W. Wernsdorfer, "From micro- to nano-SQUIDs: applications to nanomagnetism," Superconductor Science and Technology, vol. 22, no. 6, p. 064013, 2009/05/14 2009.

[4] L. Hao, "Quantum Detection Applications of NanoSQUIDs fabricated by Focussed Ion Beam," Journal of Physics: Conference Series, vol. 286, p. 012013, 2011/03/01 2011.

[5] M. J. Martínez-Pérez et al., "Three-Axis Vector Nano Superconducting Quantum Interference Device," ACS Nano, vol. 10, no. 9, pp. 8308-8315, 2016/09/27 2016.

[6] M. Schmelz et al., "3D nanoSQUID based on tunnel nano-junctions with an energy sensitivity of $1.3 \mathrm{~h}$ at 4.2 K," vol. 111, no. 3, p. 032604, 2017.

[7] M. J. Martínez-Pérez et al., "NanoSQUID Magnetometry on Individual As-grown and Annealed Co Nanowires at Variable Temperature," Nano Letters, vol. 18, no. 12, pp. 7674-7682, 2018/12/12 2018.

[8] K. K. Likharev, "Superconducting weak links," Reviews of Modern Physics, vol. 51, no. 1, pp. 101159, 01/01/ 1979.

[9] E. E. Mitchell and S. K. H. Lam, "Niobium dc SQUIDs with Nanobridge Junctions," Physics Procedia, vol. 36, pp. 382-387, 2012/01/01/ 2012.

[10] T. Godfrey, J. C. Gallop, D. C. Cox, E. J. Romans, J. Chen, and L. Hao, "Investigation of Dayem Bridge NanoSQUIDs Made by Xe Focused Ion Beam," IEEE Transactions on Applied Superconductivity, vol. 28, no. 7, pp. 1-5, 2018.

[11] L. Hao et al., "Measurement and noise performance of nano-superconducting-quantum-interference devices fabricated by focused ion beam," vol. 92, no. 19, p. 192507, 2008.

[12] O. W. Kennedy et al., "Tunable Nb Superconducting Resonator Based on a Constriction Nano-SQUID Fabricated with a Ne Focused Ion Beam," Physical Review Applied, vol. 11, no. 1, p. 014006, 01/04/ 2019.

[13] V. Bouchiat, "Detection of magnetic moments using a nano-SQUID: limits of resolution and sensitivity in near-field SQUID magnetometry," Superconductor
Science and Technology, vol. 22, no. 6, p. 064002, 2009/05/14 2009.

[14] E. M. Levenson-Falk, R. Vijay, N. Antler, and I. Siddiqi, "A dispersive nanoSQUID magnetometer for ultra-low noise, high bandwidth flux detection," Superconductor Science and Technology, vol. 26, no. 5, p. 055015, 2013/04/04 2013.

[15] S. K. H. Lam et al., "Development of a niobium nanosuperconducting quantum interference device for the detection of small spin populations," vol. 82, no. 7, pp. 1078-1080, 2003.

[16] M. Sandberg et al., "Tuning the field in a microwave resonator faster than the photon lifetime," vol. 92, no. 20, p. 203501, 2008.

[17] W. J. Skocpol, M. R. Beasley, and M. Tinkham, "Selfheating hotspots in superconducting thin-film microbridges," vol. 45, no. 9, pp. 4054-4066, 1974.

[18] D. M. Pozar, Microwave Engineering, 4th Edition. Wiley, 2011.

[19] M. Göppl et al., "Coplanar waveguide resonators for circuit quantum electrodynamics," vol. 104, no. 11, p. 113904, 2008. 